\title{
Phenotypic and genotypic determinants of mupirocin resistance among Staphylococcus aureus isolates recovered from clinical samples of children: an Iranian hospital-based study
}

This article was published in the following Dove Press journal: Infection and Drug Resistance

\author{
Shima Mahmoudi' \\ Setareh Mamishi, ${ }^{1,2}$ \\ Mohsen Mohammadi ${ }^{2}$ \\ Maryam Banar' \\ Mohammad Taghi Haghi \\ Ashtiani $^{3}$ \\ Masoumeh Mahzari² \\ Abbas Bahador ${ }^{4}$ \\ Babak Pourakbari' \\ 'Pediatric Infectious Diseases \\ Research Center, Tehran University \\ of Medical Sciences, Tehran, Iran; \\ ${ }^{2}$ Department of Infectious Disease, \\ School of Medicine, Tehran University \\ of Medical Sciences, Tehran, Iran; \\ ${ }^{3}$ Department of Pathology, School of \\ Medicine, Tehran University of Medical \\ Sciences, Tehran, Iran; ${ }^{4}$ Department \\ of Microbiology, School of Medicine, \\ Tehran University of Medical Sciences, \\ Tehran, Iran
}

Backgrounds: The aim of this study was to evaluate both phenotypic and genotypic determinants of mupirocin resistance among methicillin-resistant Staphylococcus aureus (MRSA) and methicillin susceptible $S$. aureus (MSSA) strains recovered from different clinical samples of children who were admitted to the Children's Medical Center (CMC) Hospital, Tehran, Iran. Materials and methods: A total of 120 clinical isolates of $S$. aureus were collected from the microbiology laboratory of CMC Hospital. Antimicrobial susceptibility of the isolates to different antimicrobial agents was determined by disk diffusion method. The methicillin resistance phenotype (MRSA) was identified using a $30 \mu \mathrm{g}$ cefoxitin disk. The minimum inhibitory concentration (MIC) of mupirocin was determined by broth microdilution method. Strains with mupirocin MIC between 8 and $256 \mu \mathrm{g} / \mathrm{mL}$ were considered as low-level mupirocin resistant (LLMR), and strains with an $\mathrm{MIC} \geq 512 \mu \mathrm{g} / \mathrm{mL}$ were considered as high-level mupirocin resistant (HLMR). The presence of genes encoding HLMR (ie, mupA and mupB genes) was evaluated by PCR method Results: Four out of 120 isolates (3\%) had mupirocin MIC $\geq 512 \mu \mathrm{g} / \mathrm{mL}$ and were HLMR; however, no LLMR isolate was detected. Fifty-two isolates (43\%) were MRSA, and there were no differences in the distribution of mupirocin resistance among MRSA and MSSA isolates $(P>0.05)$. The PCR method identified mupA gene in two out of four HLMR isolates, and mupB gene was not detected in any HLMR isolates.

Conclusion: Because of discrepancies between the phenotypic and genotypic patterns of mupirocin resistance and due to the avoidance of false-negative results, it is better to determine the mupirocin resistance by both antibiotic susceptibility tests and PCR method. Considering the increasing need of mupirocin for the control of $S$. aureus infections, continuous checking of its susceptibility status is necessary.

Keywords: methicillin-resistant Staphylococcus aureus, mupirocin, PCR, children

\section{Introduction}

Methicillin-resistant Staphylococcus aureus (MRSA) is a significant pathogen contributing in healthcare- and community-associated infections all over the world. ${ }^{1}$ These isolates are resistant to a wide variety of currently accessible antibacterial agents such as macrolides, lincosamides, aminoglycoside, and all beta-lactams. The occurrence of multidrug resistance (MDR) among MRSA strains is a serious dilemma in treatment and control of their infections. ${ }^{2}$

Mupirocin (pseudomonic acid A) is a topical ointment that is broadly used for the treatment of staphylococcal skin infections and is effective for nasal decolonization
Correspondence: Babak Pourakbari Pediatric Infectious Diseases Research Center, Children Medical Center Hospital School of Medicine, Tehran University of Medical Sciences, No. 62,Gharib St., Keshavarz Blvd., Tehran, Iran

Tel +982166428996

Fax +9821 66428996

Email pourakbari@razi.tums.ac.ir 
of MRSA..$^{3-5}$ The mechanism of action of mupirocin involves attaching to the bacterial isoleucyl-tRNA synthetase and interfering with protein synthesis. ${ }^{6}$ Widespread and long-term use of this medicine led to the emergence of mupirocinresistant organisms. ${ }^{7}$

According to the results of minimum inhibitory concentration (MIC) test, two kinds of mupirocin resistance have been explained. Low-level mupirocin resistance (LLMR) with an MIC between 8 and $256 \mu \mathrm{g} / \mathrm{mL}$ is mediated by point mutations in tRNA synthetase chromosomal gene (ileS-1).,6-9 This mutation is stable and nontransferable ${ }^{10}$ and its related resistance (ie, LLMR) is not clinically relevant because mupirocin is a topical antibiotic and has high concentrations on the infection site. ${ }^{11}$ High-level mupirocin resistance (HLMR) with an $\mathrm{MIC} \geq 512 \mu \mathrm{g} / \mathrm{mL}$ is the result of the acquisition of plasmid-borne resistance genes mup $A$ (also known as ileS-2) or mupB. Both the genes encode additional isoleucyl-tRNA-synthetases that are not sensitive to repression by mupirocin. ${ }^{6,10,12,13}$ The mupA encoding plasmid may also carry resistance genes to other antibiotics. So it is likely that the application of mupirocin not only results in mupirocin resistance but also leads to the increasing resistance to the other antibiotics. ${ }^{6}$

The aim of this study was to evaluate both the phenotypic and genotypic determinants of mupirocin resistance among MRSA and MSSA strains recovered from different clinical samples of children who were admitted to the Children's Medical Center (CMC) Hospital, Tehran, Iran.

\section{Materials and methods}

CMC Hospital is one of the most experienced subspecialized hospitals in Iran and offers high-quality and specialized therapeutic services to neonates, infants, and children throughout the country and region. Our center consists of about 20 specialty and subspecialty wards, including emergency medical services, electronic intensive care unit, infectious diseases, hematology, nephrology, hemodialysis, endocrinology, gastrointestinal disease, endoscopy, neonatology, neonatal intensive care unit, pediatric intensive care unit, immunology, rheumatology, neurology, psychiatry, cardiology, open heart intensive care unit, coronary intensive care unit, respiratory disease, surgery, orthopedic, cardiac, ENT, neurosurgery, urology, and operation theaters. This center was selected as the hub of excellence in pediatrics in 2008 by the Iranian Ministry of Health, Treatment and Medical Education which provides subspecialty care for more than 1,500 patients monthly.

\section{Bacterial strains}

During a cross-sectional study in 2016, a total of 120 nonduplicate clinical isolates of $S$. aureus were recovered from wound, trachea, eye, blood, inguinal region, abscess, lymph nodes, and bone marrow samples of the children admitted to CMC hospital. All of the isolates were reidentified using conventional confirmatory tests, such as Gram stain, catalase and coagulase production, DNase, and mannitol fermentation. ${ }^{14}$

\section{Antibiotic susceptibility testing}

The antibiotic susceptibility of the isolates was evaluated according to the guidelines published in 2016 by Clinical and Laboratory Standards Institute (CLSI). ${ }^{15,16}$ The following antibiotic disks were applied in disk diffusion agar test: amikacin $(30 \mu \mathrm{g})$, azithromycin $(15 \mu \mathrm{g})$, clindamycin $(2 \mu \mathrm{g})$, moxifloxacin $(5 \mu \mathrm{g})$, penicillin $(10 \mathrm{U})$, tetracycline $(30 \mu \mathrm{g})$, linezolid $(30 \mu \mathrm{g})$, cefazolin $(30 \mu \mathrm{g})$, quinupristin-dalfopristin (15 $\mu \mathrm{g})$, trimethoprim-sulfamethoxazole $(1.25 / 23.75 \mu \mathrm{g})$, chloramphenicol $(30 \mu \mathrm{g})$, minocycline $(30 \mu \mathrm{g})$, cloxacillin $(1 \mu \mathrm{g})$, cefoxitin $(30 \mu \mathrm{g})$, rifampicin $(5 \mu \mathrm{g})$, and ciprofloxacin $(5 \mu \mathrm{g})$. All disks were purchased from Mast Co., UK, and S. aureus ATCC 25923 was used for quality control of the test.

The methicillin resistance phenotype (MRSA) was identified using a $30 \mu \mathrm{g}$ cefoxitin disk, and the results were interpreted based on the CLSI standards. The MICs of vancomycin and mupirocin (Mast Co., UK) were determined by E-test and broth microdilution methods, respectively. Isolates with an $\mathrm{MIC} \leq 2 \mu \mathrm{g} / \mathrm{mL}$ were sensitive to vancomycin. Strains with their mupirocin MICs between 8 and $256 \mu \mathrm{g} / \mathrm{mL}$ were considered as LLMR, and isolates with an MIC $\geq 512 \mu \mathrm{g} / \mathrm{mL}$ were considered as HLMR.

\section{Detection of HLMR encoding genes}

Genomic DNA of HLMR isolates was extracted using the phenol-chloroform-isoamyl alcohol method as described previously. ${ }^{17}$ The mupA and mupB genes were amplified by PCR method using the following oligonucleotide primers, mupA-F: TATATTATGCGATGGAAGGTTGG, mupA-R: AATAAAATCAGCTGGAAAGTGTTG, mupBF: CTAGAAGTCGATTTTGGAGTAG, and mupB-R: AGTGTCTAAAATGATAAGACGATC.

The PCR mixture for the amplification of these genes (final volume of $25 \mu \mathrm{L}$ ) consisted of $2.5 \mu \mathrm{L}$ of reaction buffer, $0.5 \mu \mathrm{L}$ of $100 \mathrm{mM} \mathrm{MgCl}_{2}, 2.5$ units of Taq DNA polymerase, $0.5 \mu \mathrm{L}$ of $50 \mu \mathrm{M}$ dNTP, $0.5 \mu \mathrm{L}$ of $10 \mathrm{pMol}$ primer, $19.8 \mu \mathrm{L}$ of distilled water, and $0.5 \mu \mathrm{L}$ of DNA template. 
The amplification was performed using the following conditions: initial denaturation step at $94^{\circ} \mathrm{C}$ for 5 minutes, 30 cycles of $94^{\circ} \mathrm{C}$ for 30 seconds, $60^{\circ} \mathrm{C}$ for 30 seconds, and $72^{\circ} \mathrm{C}$ for 30 seconds, and a final extension at $72^{\circ} \mathrm{C}$ for 5 minutes.

\section{Statistical analysis}

Statistical analysis of the results was performed by the statistical package SPSS 16.0 (SPSS Inc. Chicago, IL, USA). Variables were compared by chi-square test and Student's $t$-test. The level of significance was set at $P<0.05$.

\section{Ethics approval and consent to participate}

The study protocol was reviewed and approved by the Pediatric Infectious Disease Research Center of Tehran University of Medical Sciences.

\section{Results}

The demographic and clinical characteristics of the patients are listed in Table 1. Fifty-six percent of the patients were male $(n=67)$ and $44 \%$ were female $(n=53)$. The wound was the most frequent site of infection $(35 \%, n=42)$, whereas the bone marrow was the least frequent site $(3 \%, n=4)$. Most of the strains $(33 \%, n=40)$ were isolated from the infectious department of the hospital and the least isolation rate was related to oncology ward $(3 \%, n=4)$.

The antibiotic susceptibility patterns of the isolates are presented in Table 2. According to the results, penicillin was

Table I Demographic and clinical data of children with Staphylococcal infection

\begin{tabular}{|l|l|}
\hline Gender & N (\%) \\
Male & $67(56)$ \\
Female & $53(44)$ \\
Specimen source & \\
Wound & $42(35)$ \\
Blood & $24(20)$ \\
Eye & $16(13)$ \\
Inguinal region & $13(11)$ \\
Trachea & $11(9)$ \\
Abscess & $5(4)$ \\
Lymph nodes & $5(4)$ \\
Bone marrow & $4(3)$ \\
Hospital ward & \\
Neonatal intensive care unit & $17(14)$ \\
Rheumatology & $8(7)$ \\
Oncology & $4(3)$ \\
Surgical & $25(21)$ \\
Pediatric intensive care unit & $10(8)$ \\
Infectious & $40(33)$ \\
Emergency & $10(8)$ \\
Gastrointestinal & $6(5)$ \\
\hline
\end{tabular}

the least effective antibiotic and had the highest resistance rate $(96 \%, \mathrm{n}=115)$. In contrast, vancomycin $(0 \%)$, amikacin $(1.66 \%, n=2)$, quinupristin-dalfopristin $(2 \%, n=2)$, rifampicin $(2 \%, n=2)$, linezolid $(3 \%, n=4)$, and cefazolin $(10 \%$, $\mathrm{n}=12$ ) were the most effective antibiotics. Four out of 120 isolates $(3 \%)$ were resistant to mupirocin, and their MIC was $\geq 512 \mu \mathrm{g} / \mathrm{mL}$ (ie, HLMR) and no LLMR isolate was detected (Table 3).

A total of 52 isolates (43\%) were resistant to methicillin (MRSA). There were significant statistical differences between the resistance rates of MRSA and methicillin sensitive (MSSA) isolates to amikacin, cefazolin, penicillin, cloxacillin, and ciprofloxacin $(P<0.05)$; and MRSA isolates showed higher rates. However, there were no significant statistical differences between the resistance rates of these two groups against other tested antibiotic $(P>0.05)$ (Table 3$)$. Vancomycin (0\%), linezolid (2\%), mupirocin (4\%), quinupristin-dalfopristin (6\%), and rifampicin (6\%) were the most effective antibiotics against MRSA isolates. There were no differences in the distribution of mupirocin resistance among MRSA and MSSA isolates $(P>0.05)$.

The PCR method identified mupA (ileS-2) gene in two out of four HLMR isolates, and mupB gene was not detected in any HLMR isolates.

\section{Discussion}

S. aureus is an important human pathogen responsible for a wide range of infections such as skin and soft tissue infections, endovascular infections, pneumonia, septic arthritis, endocarditis, osteomyelitis, foreign-body infections, and sepsis. ${ }^{18,19}$ Administration of mupirocin is an old preventive method for eradication of $S$. aureus carriage in patients undergoing surgery or dialysis and patients hospitalized in intensive care units. ${ }^{6}$ Likewise, the use of mupirocin is a common approach for decolonization of nasal MRSA and control of its spread in hospitals. ${ }^{10}$ It is also a good choice for the treatment of mild skin infections caused by MRSA strains. ${ }^{20}$ However, the emergence of resistance to this drug limits its efficiency. In this study, the prevalence of both phenotypic and genotypic determinants of mupirocin resistance among MRSA and MSSA strains retrieved from children with a variety of staphylococcal infections was evaluated.

In this study, the isolation rate of $S$. aureus was higher in male patients (male to female ratio: 1.2). This was similar to the rate observed in the previous study conducted in CMC hospital. ${ }^{21}$ In contrast to our study that wound was the most common isolation site $(35 \%)$, in the study conducted by 
Table 2 The antibiotic susceptibility patterns of Staphylococcus aureus isolates

\begin{tabular}{|l|l|l|l|}
\hline Antibiotic & Sensitive N (\%) & Intermediate N (\%) & Resistant N (\%) \\
\hline Amikacin & $110(91.66)$ & $8(6.66)$ & $2(1.66)$ \\
Azithromycin & $52(43)$ & $3(3)$ & $65(54)$ \\
Moxifloxacin & $80(77)$ & $2(2)$ & $22(21)$ \\
Tetracycline & $90(75)$ & - & $30(25)$ \\
Vancomycin & $120(100)$ & - & - \\
Cefazolin & $108(90)$ & - & $12(10)$ \\
Trimethoprim-sulfamethoxazole & $86(72)$ & - & $34(28)$ \\
Quinupristin-dalfopristin & $114(95)$ & $4(3)$ & $2(2)$ \\
Chloramphenicol & $84(70)$ & $29(24)$ & $7(6)$ \\
Clindamycin & $67(56)$ & $33(27.5)$ & $20(16.5)$ \\
Penicillin & $5(4)$ & - & $115(96)$ \\
Linezolid & $116(97)$ & - & $4(3)$ \\
Minocycline & $103(86)$ & $4(3)$ & $13(11)$ \\
Cloxacillin & $83(69)$ & - & $37(31)$ \\
Methicillin & $68(57)$ & - & $52(43)$ \\
Rifampicin & $111(92)$ & $3(6)$ & $2(2)$ \\
Ciprofloxacin & $79(77)$ & & $21(20)$ \\
\hline
\end{tabular}

Table 3 The antibiotic susceptibility patterns of Staphylococcus aureus isolates

\begin{tabular}{|c|c|c|c|c|}
\hline \multirow[t]{2}{*}{ Antibiotic } & \multicolumn{2}{|c|}{$N(\%)$ of MSSA $(n=68)$} & \multicolumn{2}{|c|}{$N(\%)$ of MRSA $(n=52)$} \\
\hline & Sensitive & Resistant & Sensitive & Resistant \\
\hline Amikacin & $68(100)$ & $0(0)$ & $42(8 I)$ & $10(19)$ \\
\hline Azithromycin & $31(46)$ & $37(54)$ & $21(40)$ & $31(60)$ \\
\hline Moxifloxacin & $55(81)$ & $13(19)$ & $41(79)$ & $\mathrm{II}(2 \mathrm{I})$ \\
\hline Tetracycline & $51(75)$ & $17(25)$ & $39(75)$ & $13(25)$ \\
\hline Vancomycin & $68(100)$ & $0(0)$ & $52(100)$ & $0(0)$ \\
\hline Cefazolin & $68(100)$ & $0(0)$ & $40(77)$ & $12(23)$ \\
\hline Trimethoprim-sulfamethoxazole & $53(78)$ & $15(22)$ & $33(63.5)$ & $19(36.5)$ \\
\hline Quinupristin-dalfopristin & $65(96)$ & $3(4)$ & $49(94)$ & $3(6)$ \\
\hline Chloramphenicol & $49(72)$ & $19(28)$ & $35(67)$ & $17(33)$ \\
\hline Clindamycin & $36(53)$ & $32(47)$ & $31(60)$ & $21(40)$ \\
\hline Penicillin & $5(7)$ & $63(93)$ & $0(0)$ & $52(100)$ \\
\hline Linezolid & $65(96)$ & $3(4)$ & $51(98)$ & $\mathrm{I}(2)$ \\
\hline Minocycline & $60(88)$ & $8(12)$ & $43(83)$ & $9(17)$ \\
\hline Cloxacillin & $61(90)$ & $7(10)$ & $22(42)$ & $30(58)$ \\
\hline Mupirocin & $66(97)$ & $2(3)$ & $50(96)$ & $2(4)$ \\
\hline Rifampicin & $64(94)$ & $4(6)$ & $49(94)$ & $3(6)$ \\
\hline Ciprofloxacin & $59(87)$ & $9(13)$ & 37 (7I) & $15(29)$ \\
\hline
\end{tabular}

Sabouni et al, ${ }^{21}$ highest isolation rate was related to jaundice $(12 \%)$, and $8 \%$ of strains were isolated from wound infection.

In the current study, the highest antibiotic resistance rate was against penicillin (96\%), but vancomycin, amikacin, quinupristin-dalfopristin, rifampicin, linezolid, and cefazolin had the least resistance rates. In the study performed by Jung et al, ${ }^{22}$ from Korea, similar resistance rates against vancomycin, linezolid, quinupristin-dalfopristin, clindamycin, and rifampicin was found. However, resistance rates of trimethoprim-sulfamethoxazole, tetracycline, and ciprofloxacin were lower than our study. In addition, Cavalcant et al, from Brazil ${ }^{23}$ reported lower rates of resistance for ciprofloxacin, tetracycline, and trimethoprim-sulfamethoxazole. The acquisition of mobile genetic elements (MGEs) harboring multiresistance genes can be a possible reason for higher resistance rates of trimethoprim-sulfamethoxazole, tetracycline, and ciprofloxacin that were observed in this study. ${ }^{24}$

In the present study, HLMR was identified in four isolates $(3 \%)$. Heterogeneity in mupirocin resistance in different studies has been reported. This rate differs according to the characteristics of studied patients, origin of isolates, and geographical areas. ${ }^{25}$ In the study conducted by Pereira 
et al, in Brazil, ${ }^{11}$ and Emaneini et al, in Iran, ${ }^{26}$ none of the tested isolates $(0 \%)$ were resistant to mupirocin. In the study performed by Hogue et al, in northwest USA, ${ }^{3} 1.8 \%$ of the studied population were colonized with HLMR $S$. aureus. However, in other studies from USA, ${ }^{9,20}$ relative high resistance rates were reported ( $14.7 \%$ and $31.3 \%$, respectively). In view of some investigators, there is a strong correlation between the emergence of mupirocin resistance and previous consumption of this drug. ${ }^{4,8,20,27,28}$ The low rate of mupirocin resistance observed in this study could be due to the lesser exposure of patients to this antibiotic.

According to the results, $43 \%(n=52)$ of our isolates were MRSA which is near to the rate of our previous study $(48 \%)^{29}$ and higher than that reported from Korea (19\%), ${ }^{22}$ Brazil $(26.6 \%),{ }^{23}$ and Iran (11.5\%). ${ }^{26}$ The rates of MRSA isolated from blood cultures in our previous study $(79 \%)^{30}$ and the rate reported by McNeil et al from USA $(79.5 \%)^{9}$ were higher than this study. A reason for discrepancies in rates of MRSA could be the diverse source of investigated $S$. aureus isolates in each study and differences in infection control strategies in different regions of the world. ${ }^{26}$

In the current study, there were significant differences in the resistance rates to cefazolin, penicillin, cloxacillin, ciprofloxacin, and amikacin between MRSA and MSSA isolates, and MRSA isolates presented higher rates. There are other reports about higher antibiotic resistance rates of MRSA in comparison with MSSA isolates. ${ }^{30,31}$ MRSA isolates possess an MGE called staphylococcal cassette chromosome mec, which carries methicillin resistance gene (ie, mecA) and several other genes conferring resistance to non-beta-lactam antibiotics and is in charge of MDR in MRSA isolates. ${ }^{32}$ This phenomenon results in the diminished efficacy of most antimicrobial agents in the eradication of MRSA infections and can complicate the selection of proper therapeutic regimens for them..$^{33,34}$

Fortunately, in this survey, the rate of mupirocin resistance in MRSA isolates was low (4\%) and was similar to the resistance rate of MSSA isolates (Table 3). However, in the study performed by Baek et al, ${ }^{33}$ there were significant differences between the mupirocin resistance rates in MRSA and MSSA isolates $(12.7 \%$ vs $4.5 \%$, respectively). Furthermore, in the study conducted by Antonov et al, ${ }^{20}$ the rate of mupirocin resistance was higher among MRSA isolates and MRSA was recognized as a risk factor for resistance to this antibiotic. In contrary, McNeil et al, ${ }^{9}$ observed more mupirocin resistance among MSSA isolates. With regard to the limited therapeutic options for MRSA, continuous monitoring of the emergence of antibiotic resistance in these pathogens (especially against mupirocin) and ensuring the usefulness of the treatment regimens is also important. ${ }^{3,11}$

The genotypic and phenotypic characteristics of mupirocin resistance were consistent in two HLMR isolates. However, for two other isolates, there were discrepancies between MIC and PCR results. In the study performed by McNeil et al, ${ }^{9}$ similar results were observed, and there were two strains with HLMR phenotype which did not harbor mupA gene. In contrast, in the study conducted by Wang et al, ${ }^{1}$ mupA gene was detected in all of the HLMR isolates. In the study performed by Hesami et al, ${ }^{35}$ mupA gene was detected in ten out of eleven mupirocin-resistant isolates and one isolate possessed iles- 1 gene, and mupB gene was not detected in any of the strains.

In the study of McNeil et al, two mupA-negative isolates were HMR. Although sequencing of both the isolates was performed, no mutations in ileS-1 gene were observed. Therefore, it is possible that these isolates acquired HMR by another unknown mechanism. ${ }^{9}$

One of the limitations of this study is the lack of information about the prior use of mupirocin by patients. Therefore, the impact of previous consumption of mupirocin on the emergence of resistance against this drug cannot be evaluated. Another limitation of our study is that the presence of iles-1 gene (which is responsible for LLMR in isolates of $S$. aureus) and possible mutations in this gene was not detected. The reason for HLMR in the two mup $\mathrm{A}^{-} / m u p \mathrm{~B}^{-}$was unknown. Further studies about the molecular basis of HLMR in the two mup $A^{-} /$mup $B^{-}$isolates are continued.

\section{Conclusion}

Due to the low resistance rates of MRSA isolates against vancomycin, linezolid, mupirocin, quinupristin-dalfopristin, and rifampicin in this study, it is suggested that these antibiotics should be used in empirical treatment of intricate infections caused by MRSA. With a view to the disparate results of antibiotic susceptibility testing and PCR for designation of mupirocin resistance, it is recommended that determination of mupirocin resistance cannot be done only on the basis of PCR method and this can increase the amount of false-negative results. Despite the low resistance rate to mupirocin $(3 \%)$ and vancomycin $(0 \%)$, these drugs should be prescribed cautiously to reserve important drugs in controlling MRSA infections. Regular monitoring of the usage of mupirocin and the rate of its resistance among MRSA isolates is necessary. 


\section{Acknowledgments}

This study was Dr Mohsen Mohammadi's postgraduate thesis and was supported by a grant (grant number: 93-0388-21823) from Tehran University of Medical Sciences to Dr Babak Pourakbari. The abstract of this paper was presented at the 18th International and Iranian Congress of Microbiology as a conference talk with interim findings (http://health. barakatkns.com/seminar-article/464470/phenotypic-andgenotypic-determinants-of-mupirocin-resistance-amongstaphylococcus-aureus-isolates-r).

\section{Disclosure}

The authors report no conflicts of interest in this work.

\section{References}

1. Wang L, Liu Y, Yang Y, et al. Multidrug-resistant clones of communityassociated methicillin-resistant Staphylococcus aureus isolated from Chinese children and the resistance genes to clindamycin and mupirocin. J Med Microbiol. 2012;61(9):1240-1247.

2. Goudarzi M, Seyedjavadi SS, Nasiri MJ, Goudarzi H, Sajadi Nia R, Dabiri H. Molecular characteristics of methicillin-resistant Staphylococcus aureus (MRSA) strains isolated from patients with bacteremia based on MLST, SCCmec, spa, and agr locus types analysis. Microb Pathog. 2017;104:328-335.

3. Hogue JS, Buttke P, Braun LE, Fairchok MP. Mupirocin resistance related to increasing mupirocin use in clinical isolates of methicillinresistant Staphylococcus aureus in a pediatric population. J Clin Microbiol. 2010;48(7):2599-2600.

4. Lee AS, Macedo-Vinas M, François P, et al. Trends in mupirocin resistance in methicillin-resistant Staphylococcus aureus and mupirocin consumption at a tertiary care hospital. J Hosp Infect. 2011;77(4):360-362.

5. Shahsavan S, Emaneini M, Noorazar Khoshgnab B, et al. A high prevalence of mupirocin and macrolide resistance determinant among Staphylococcus aureus strains isolated from burnt patients. Burns. 2012;38(3):378-382.

6. Fritz SA, Hogan PG, Camins BC, et al. Mupirocin and chlorhexidine resistance in Staphylococcus aureus in patients with communityonset skin and soft tissue infections. Antimicrob Agents Chemother. 2013;57(1):559-568.

7. Nakajima J, Hitomi S, Kurihara Y. Detection of methicillin-resistant Staphylococcus aureus with high-level resistance to mupirocin. J Infect Chemother. 2011;17(6):868-871.

8. Caffrey AR, Quilliam BJ, Laplante KL. Risk factors associated with mupirocin resistance in methicillin-resistant Staphylococcus aureus. $J$ Hosp Infect. 2010;76(3):206-210.

9. Mcneil JC, Hulten KG, Kaplan SL, Mason EO. Mupirocin resistance in Staphylococcus aureus causing recurrent skin and soft tissue infections in children. Antimicrob Agents Chemother. 2011;55(5):2431-2433

10. Hayden MK, Lolans K, Haffenreffer K, et al. Chlorhexidine and mupirocin susceptibility of methicillin-resistant staphylococcus aureus isolates in the REDUCE-MRSA trial. J Clin Microbiol. 2016;54(11): 2735-2742.

11. Pereira MF, Berezin EN, Carvalho RL, Scheffer DK, Mimica MJ. Mupirocin susceptibility in Staphylococcus aureus nasal and oropharyngeal isolates from Brazilian children. J Chemother. 2014;26(6):373-374.

12. Joshi PR, Acharya M, Aryal R, et al. Emergence of staphylococcal cassette chromosome mec type I with high-level mupirocin resistance among methicillin-resistant Staphylococcus aureus. Asian Pac J Trop Biomed. 2017;7(3):193-197.
13. Bathoorn E, Hetem DJ, Alphenaar J, Kusters JG, Bonten MJ. Emergence of high-level mupirocin resistance in coagulase-negative staphylococci associated with increased short-term mupirocin use. J Clin Microbiol. 2012;50(9):2947-2950.

14. Mahon CR, Lehman DC, Manuselis G Jr. Textbook of Diagnostic Microbiology. Toronto: Elsevier Health Sciences; 2014.

15. Wayne P. Methods for Dilution Antimicrobial Susceptibility Tests for Bacteria That Grow Aerobically. Approved standard 10th edition. Clinical and Laboratory Standards Institute, CLSI document M07-A10; 2015. Available from: https://clsi.org/standards/products/microbiology/ documents $/ \mathrm{m} 07 /$. Accessed.

16. Wayne P. Performance Standards for Antimicrobial Susceptibility Testing. 28th edition. Clinical and Laboratory Standards Institute, CLSI supplement M100S; 2018. Available from: https://clsi.org/standards/ products/microbiology/documents/m100/. Accessed.

17. Gomroki F, Mohammed HB, Malla S. Amplification of Methicillin Resistant Gene (mecA) gene from the MRSA strains. Int J Pharm Clin Res; 2015;7(3):198-203.

18. Benito D, Aspiroz C, Gilaberte Y, et al. Genetic lineages and antimicrobial resistance genotypes in Staphylococcus aureus from children with atopic dermatitis: detection of clonal complexes CC1, CC97 and CC398. J Chemother. 2016;28(5):359-366.

19. David MZ, Daum RS. Community-associated methicillin-resistant Staphylococcus aureus: epidemiology and clinical consequences of an emerging epidemic. Clin Microbiol Rev. 2010;23(3):616-687.

20. Antonov NK, Garzon MC, Morel KD, Whittier S, Planet PJ, Lauren CT. High prevalence of mupirocin resistance in Staphylococcus aureus isolates from a pediatric population. Antimicrob Agents Chemother. 2015;59(6):3350-3356.

21. Sabouni F, Ranjbari R, Pourakbari B, et al. Staphylococcus aureus infections in children in an Iranian referral pediatric hospital. J Prev Med Hyg. 2013;54(4):205.

22. Jung MY, Chung JY, Lee HY, Park J, Lee DY, Yang JM. Antibiotic susceptibility of staphylococcus aureus in atopic dermatitis: current prevalence of methicillin-resistant staphylococcus aureus in Korea and treatment strategies. Ann Dermatol. 2015;27(4):398-403.

23. Cavalcante FS, Abad ED, Lyra YC, et al. High prevalence of methicillin resistance and PVL genes among Staphylococcus aureus isolates from the nares and skin lesions of pediatric patients with atopic dermatitis. Braz J Med Biol Res. 2015;48(7):588-594.

24. Han LL, Mcdougal LK, Gorwitz RJ, et al. High frequencies of clindamycin and tetracycline resistance in methicillin-resistant Staphylococcus aureus pulsed-field type USA300 isolates collected at a Boston ambulatory health center. J Clin Microbiol. 2007;45(4):1350-1352.

25. Nejabat M, Khashei R, Bazargani A, Sedigh Ebrahim-Saraie H, Motamedifar M. Evaluation of high-level of mupirocin resistance among clinical isolates of methicillin-resistant staphylococcus aureus from shiraz, Iran (2008-2009). Pharmaceutical Sciences. 2015;21(4): 225-228.

26. Emaneini M, Khoramrooz SS, Taherikalani M, Jabalameli F, Aligholi M. Molecular characterization of Staphylococcus aureus isolated from children with adenoid hypertrophy: emergence of new spa types $\mathrm{t} 7685$ and t7692. Int J Pediatr Otorhinolaryngol. 2011;75(11):1446-1449.

27. Mcneil JC, Hulten KG, Kaplan SL, Mason EO. Decreased susceptibilities to Retapamulin, Mupirocin, and Chlorhexidine among Staphylococcus aureus isolates causing skin and soft tissue infections in otherwise healthy children. Antimicrob Agents Chemother. 2014;58(5): 2878-2883.

28. Talon D, Marion C, Thouverez M, Bertrand X. Mupirocin resistance is not an inevitable consequence of mupirocin use. J Hosp Infect. 2011;79(4):366-367.

29. Mamishi S, Mahmoudi S, Sadeghi RH, Movahedi Z, Hadipour R, Pourakbari B. Genotyping of Staphylococcus aureus strains among healthcare workers and patients in the tertiary referral Children's Medical Hospital in Tehran, Iran. Br J Biomed Sci. 2012;69(4):173. 
30. Pourakbari B, Sadr A, Ashtiani MTH. Five-year evaluation of the antimicrobial susceptibility patterns of bacteria causing bloodstream infections in Iran. J Infect Develop Countries. 2011;6(2): $120-125$.

31. Wu D, Wang Q, Yang Y, et al. Epidemiology and molecular characteristics of community-associated methicillin-resistant and methicillinsusceptible Staphylococcus aureus from skin/soft tissue infections in a children's hospital in Beijing, China. Diagn Microbiol Infect Dis. 2010;67(1):1-8.

32. Zetola N, Francis JS, Nuermberger EL, Bishai WR. Communityacquired meticillin-resistant Staphylococcus aureus: an emerging threat. Lancet Infect Dis. 2005;5(5):275-286.
33. Baek YS, Jeon J, Ahn JW, Song HJ. Antimicrobial resistance of Staphylococcus aureus isolated from skin infections and its implications in various clinical conditions in Korea. Int J Dermatol. 2016;55(4):e191-e197.

34. Ghadiri K, Novroozi B, Rezaee M. Comparison of Mec a gene in staphylococcus aureus isolated from children at time of admission and discharge in West of Iran. International Journal of Medical Research \& Health Sciences. 2016;5(5):133-139.

35. Hesami S, Hosseini SD, Amouzandeh-Nobaveh A, Eskandari S, Ghaznavi-Rad E. Phenotypic and genotypic determination of mupirocin resistance among methicillin susceptibility and resistance in staphylococci isolated from nosocomial infections. Journal of Mazandaran University of Medical Sciences. 2014;23(1):30-39.
Infection and Drug Resistance

\section{Publish your work in this journal}

Infection and Drug Resistance is an international, peer-reviewed openaccess journal that focuses on the optimal treatment of infection (bacterial, fungal and viral) and the development and institution of preventive strategies to minimize the development and spread of resistance. The journal is specifically concerned with the epidemiology of antibiotic

\section{Dovepress}

resistance and the mechanisms of resistance development and diffusion in both hospitals and the community. The manuscript management system is completely online and includes a very quick and fair peerreview system, which is all easy to use. Visit http://www.dovepress.com/ testimonials.php to read real quotes from published authors.

Submit your manuscript here: https://www.dovepress.com/infection-and-drug-resistance-journal 